\title{
Magnetic anisotropy and core-shell origin of the biogenic ferrihydrite nanoparticles
}

Yu.V. Knyazev ${ }^{1}$, D.A. Balaev ${ }^{1,2}$, S.V. Stolyar ${ }^{2,3}$, O.A. Bayukov ${ }^{1}$, R.N. Yaroslavtsev ${ }^{1,3}$, V.P.Ladygina ${ }^{3}$, D.A. Velikanov ${ }^{1}$, R.S. Iskhakov ${ }^{1}$

1 - Kirensky Institute of Physics, Federal Research Center KSC SB RAS, Krasnoyarsk, Russia

2 - Siberian Federal University, Krasnoyarsk, Russia

3 - Krasnoyarsk Scientific Center, Federal Research Center KSC SB RAS, Krasnoyarsk, Russia

\begin{abstract}
Ferrihydrite is a mineral that exists in a nanoscale form. At the antiferromagnetic ordering of the magnetic moments of iron atoms, it exhibits, due to the uncompensated magnetic moment of its nanoparticles, the magnetic properties very similar to those of ferro- and ferrimagnetic nanoparticles. In this study, we investigate the ferrihydrite nanoparticles that are a co-product of the cultivation of bacteria. The obtained biogenic sample is an aggregate of biogenic nanoparticles with an average size of $\sim 2 \mathrm{~nm}$ coated with a polysaccharide shell, which excludes contact magnetic interactions between particles. The features caused by the surface effects and the inhomogeneous structure of ferrihydrite have been examined in the temperature range of 4-300 K using Mössbauer spectroscopy and magnetometry. Based on the data obtained, the superparamagnetic blocking processes have been identified. The dominating contribution of the surface magnetic anisotropy to these processes has been established. This surface effect is caused by the inhomogeneous structure of ferrihydrite nanoparticles, which is confirmed by the Mössbauer spectroscopy data showing the existence of different states of iron nuclei. A core-shell structural model of ferrihydrite particles has been proposed. The particle core is well-crystallized, while the surface layer is loose and contains a greater number of defects. It has been found that the size of the dense core depends on the particle size; the critical value is $d \sim 2$ $\mathrm{nm}$ and the particles with a smaller diameter have no dense core. The results obtained reveal inhomogeneities of the crystal structure in ferrihydrite nanoparticles, which affect the magnetic properties of the investigated materials.
\end{abstract}

\section{Introduction}

Ferrihydrite plays a decisive role in the circulation of iron and controls the iron concentration in aqueous systems. This mineral with the nominal formula $\mathrm{Fe}_{2} \mathrm{O}_{3} \cdot \mathrm{nH}_{2} \mathrm{O}$ precipitates as a product of nucleation of hydrolyzed ferric ions with increasing $\mathrm{pH}$ [1]. The discovery of ferrihydrite on Mars [2, 3] indirectly confirmed the presence of water on this planet. The size of crystallized ferrihydrite particles is no more than $\sim 10 \mathrm{~nm}$ [2]. A huge surface makes ferrihydrite a good sorbent of heavy metals. This mineral is of crucial importance for microorganisms' life [4]. Ferrihydrite can form also in the bacterial life cycle [5]. This mineral is involved in the vital activity of higher animals, including humans. In living organisms, ferrihydrite forms in the core of a ferritin complex consisting of a protein shell capsule with the ordered hydrated iron oxide core. In the most well-studied horse spleen ferritin, the ordered ferrihydrite core is located inside the protein shell with outer and inner diameters of 12 and 
5-8 nm, respectively [7]. It is noteworthy that the organic shell makes the magnetic interparticle interactions negligible [8].

The conditions for the formation of ferrihydrite significantly affect the physical properties of its nanoparticles, concerning particularly its crystallinity and interactions between individual particles [9-11]. The crystal structure of ferrihydrite and its chemically synthesized aggregates was studied in $[12,13]$. The obtained X-ray data made it possible not only to refine the crystal structure and chemical formula of ferrihydrite, but also to determine the degree of its atomic disorder. Study of aggregation of ferrihydrite nanocrystals showed that the formation of aggregates cannot be described by conventional growth models [14]. A nanosized ferrihydrite particle can be presented by a model with different surface (shell) and internal (core) positions of iron atoms [15]. As was mentioned in [16], the structure of ferrihydrite polyhedra near the surface, which is depleted in iron and rich in $\mathrm{OH}$ groups, strongly differs from the structure of the particle core [16]. As a result of such conditions of the particle surface formation, the enthalpy of the formation of the ferrihydrite surface is lower than the bulk enthalpy as compared with other iron oxides and hydroxides [7]. In this case, the particle itself remains chemically stable. The established effect of depletion of the nanoparticle surface [16] manifests itself in the low nanoparticle fractal dimension ( 2.82) determined by the small-angle X-ray scattering technique [17, $18]$.

The magnetic moments of iron atoms in ferrihydrite are antiferromagnetically ordered; according to the neutron diffraction data, the Néel temperature of the material is 350 K [19]. In turn, the imperfection of the ferrihydrite crystal structure leads to the occurrence of an uncompensated magnetic moment in nanoparticles [20-26]. This fact subsumes ferrihydrite to the class of antiferromagnetic (AFM) nanoparticles [8, 27]. The uncompensated magnetic moment of AFM nanoparticles determines their magnetic properties, which are very similar to those of single-domain ferromagnetic (FM) and ferrimagnetic nanoparticles [8, 19, 20-27]. In AFM nanoparticles, the magnetic properties exhibit the features typical of those of ordinary FM nanoparticles, including the transition from the unblocked or superparamagnetic (SP, in case of the uncompensated magnetic moments of particles) state to the blocked state with a decrease in temperature (or increase in size at $\mathrm{T}$ $=$ const $)$ and the dependence of the SP blocking temperature on the ratio between the characteristic particle relaxation time and the detection time determined by a measurement procedure used. The uncompensated magnetic moment of AFM nanoparticles can attain several hundreds of Bohr magnetons [20-35], which is comparable with the magnetic moment of FM oxide particles several nanometers in size [36-38]. The latter opens wide prospects for use of AFM nanoparticles, owing to their magnetization impacted by their nanometer dimensions. Possible medical applications of ferrihydrite based on its magnetic properties were described in [39, 40]. 
The combination of the above-mentioned factors (AFM ordering, uncompensated magnetic moment, developed surface, surface depletion effect $[15,16]$, and possible magnetic interparticle interactions [41-43]) determines a fairly complex behavior of the magnetic properties of ferrihydrite. Nevertheless, a number of bright effects directly reflecting the core-shell structure of ferrihydrite nanoparticles were established using the quasi-static magnetization [22, 24] and ferromagnetic resonance [44] measurements. In this work, we use Mössbauer spectroscopy to study the features of the manifestation of magnetism of surface and internal iron atoms in biogenic ferrihydrite nanoparticles. To unambiguously separate the contributions of the core-shell structure and the effect of the transition to the SP state, we analyze the temperature evolution of the Mössbauer spectra and temperature dependences of the quasi-static magnetization. In the investigated biogenic ferrihydrite, nanoparticles (nanocrystals) have a polysaccharide shell [45]. This allows us to ignore the magnetic interactions between particles from the analysis, as in the case of horse spleen ferritin.

\section{Experimental}

The Klebsiella oxytoca strain used was extracted from the sapropel of the Borovoe Lake, Krasnoyarsk Territory, Russia. Microorganisms were seeded on an agarized medium and grown under anaerobic conditions [46, 47]. After the repeated ultrasonic treatment of precipitated bacteria, centrifugation, and washing, a stable nanoparticle sol in an aqueous solution was prepared and then dried. The obtained nanoparticle powder was the object of study.

The electron microscopy and microdiffraction investigations were carried out on a Hitachi HT7700 transmission electron microscope at an accelerating voltage of $100 \mathrm{kV}$. Specimens were prepared by shaking the nanoparticle powder in alcohol in an ultrasonic bath and depositing the obtained suspension onto support meshes with a perforated carbon coating.

The temperature dependences of the magnetization $\mathrm{M}(\mathrm{T})$ were measured on a SQUID magnetometer [48] in the zero external field cooling (ZFC) and field cooling (FC) modes.

The Mössbauer spectra of the investigated samples were obtained on an MS-1104Em spectrometer (Research Institute of Physics, Southern Federal University) in the transmission geometry with a $\mathrm{Co}^{57}(\mathrm{Rh})$ radioactive source in the temperature range of 4-300 $\mathrm{K}$ using a CFSG-311-MESS cryostat with a sample in the exchange gas based on a closed-cycle Gifford-McMahon cryocooler (Cryotrade Engineering). The spectra were processed by varying the entire set of hyperfine parameters by the least squares method in the linear approximation. The spectra were simulated by Lorentz lines with regard to the broadenings caused by the magnetic and crystalline inhomogeneity of the samples.

\section{Results and Discussion}

\subsection{Microstructure}


Figure 1a shows typical transmission electron microscopy (TEM) data for the obtained nanoparticles and their size distribution $f(\mathrm{~d})$. The average particle diameter $<\mathrm{d}>$ was found to be $2 \mathrm{~nm}$. Figure $1 \mathrm{~b}$ presents a microdiffraction pattern characteristic of ferrihydrite nanoparticles [49-51]. One can see two diffuse reflections with interplanar spacings of 1.5 and $2.6 \AA$.

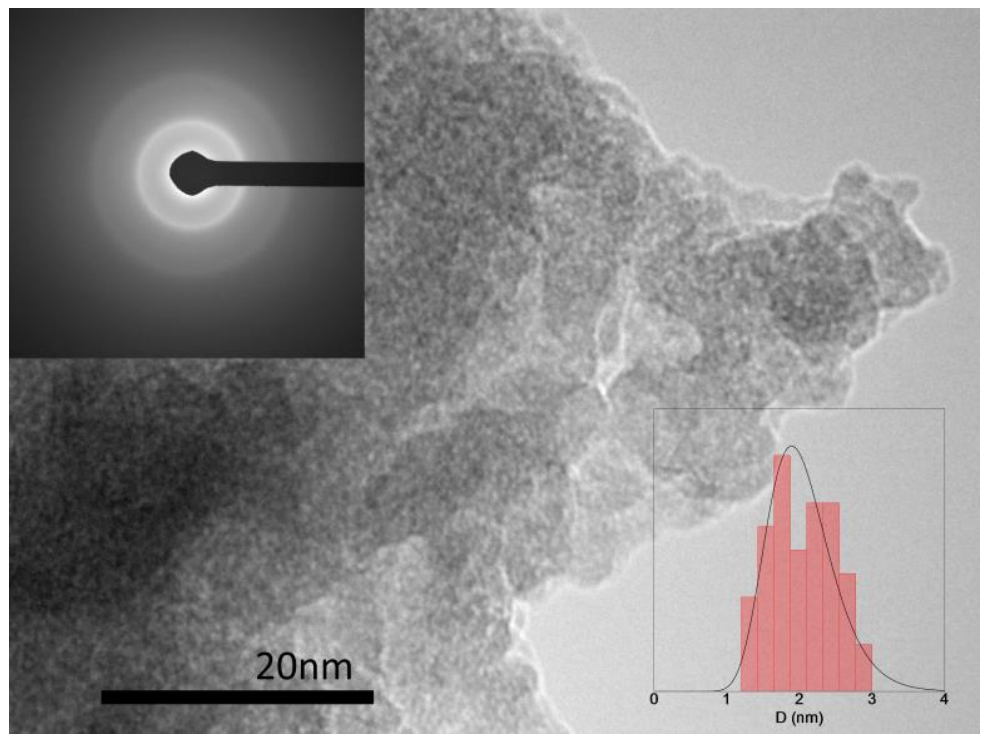

Fig. 1. TEM image of biogenic ferrihydrite nanoparticles. Inset: particle size distribution and microdiffraction pattern.

\subsection{SP Blocking for the Magnetic Measurements and Mössbauer Spectroscopy}

Figure $2 \mathrm{a}$ shows the $\mathrm{M}(\mathrm{T})$ dependences of the investigated ferrihydrite nanoparticle sample that were recorded in the $\mathrm{ZFC}$ mode and in the $\mathrm{FC}$ mode in fields of $1 \mathrm{Oe}$ and $1 \mathrm{kOe}$. The $\mathrm{M}(\mathrm{T})_{\mathrm{ZFC}}$ dependences contain pronounced maxima, which shift toward lower temperatures $\left(\mathrm{T}_{\max } \approx 13.7 \mathrm{~K}\right.$ at $\mathrm{H}=1 \mathrm{Oe}$ and $\sim 9.5 \mathrm{~K}$ at $\mathrm{H}=1 \mathrm{kOe}$ ) with increasing field. Near $\mathrm{T}_{\max }$, the $\mathrm{M}(\mathrm{T})_{\mathrm{ZFC}}$ and $\mathrm{M}(\mathrm{T})_{\mathrm{FC}}$ dependences start diverging; the divergence increases with decreasing temperature. This behavior is typical of the SP blocking processes.

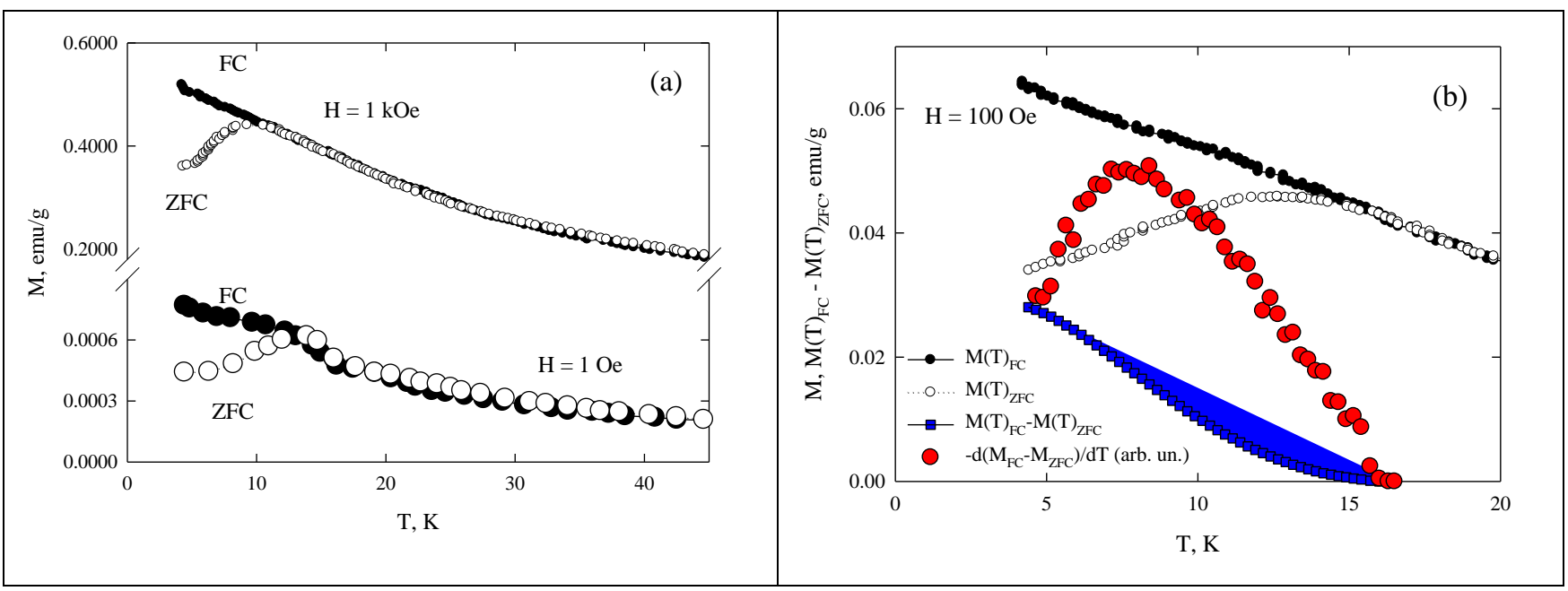


Fig. 2. Temperature dependences of magnetization measured under ZFC and FC conditions in fields of $1 \mathrm{Oe}, 1 \mathrm{kOe}(\mathrm{a})$, and $100 \mathrm{Oe}(\mathrm{b})$. In (b) also plotted the difference $\mathrm{M}(\mathrm{T})_{\mathrm{FC}}-\mathrm{M}(\mathrm{T})_{\mathrm{ZFC}}$ and the derivative $\left(\mathrm{d}\left(\mathrm{M}(\mathrm{T})_{\mathrm{FC}}-\mathrm{M}(\mathrm{T})_{\mathrm{ZFC}}\right) / \mathrm{dT}\right.$.

Figure $2 \mathrm{~b}$ presents the $\mathrm{M}(\mathrm{T})$ dependences recorded in the ZFC mode and in the FC mode in a field of 100 Oe. It is well-known that the derivative $\left(\mathrm{d}\left(\mathrm{M}(\mathrm{T})_{\mathrm{FC}}-\mathrm{M}(\mathrm{T})_{\mathrm{ZFC}}\right) / \mathrm{dT}\right.$ reflects the blocking temperature distribution function $f\left(\mathrm{~T}_{\mathrm{B}}\right)$, the maximum of which can be taken as an average value $\left\langle\mathrm{T}_{\mathrm{B}}\right\rangle$ [52-54]. The $\left(\mathrm{M}(\mathrm{T})_{\mathrm{FC}}-\mathrm{M}(\mathrm{T})_{\mathrm{ZFC}}\right)$ dependence and its derivative $f\left(\mathrm{~T}_{\mathrm{B}}\right)$ are shown in Fig. 2b. Below, we use these data to make a comparison with the $f(\mathrm{~d})$ dependence based on the TEM results.

The Mössbauer spectra of the investigated biogenic ferrihydrite sample are shown in Fig. 3. At room temperature, the spectrum is a quadrupole doublet, which is explained by the SP behavior of ferrihydrite nanoparticles [1, 51, 54-57]. The room-temperature spectrum reveals three nonequivalent positions of ferric iron in the octahedral environment, which are characteristic of ferrihydrite; their parameters are given in Table 1. At a temperature of $30 \mathrm{~K}$, a sextet arises in the spectrum. This temperature is much lower than the temperature of the AFM ordering of ferrihydrite; therefore, the occurrence of the magnetically split component in the Mössbauer spectrum can be considered in terms of blocking of the magnetic moments of particles (the uncompensated moments for AFM nanoparticles) [58]. Obviously, $30 \mathrm{~K}$ is the blocking temperature of the largest particles. With decreasing temperature, the SP fractions and the magnetically split parts of the spectrum are redistributed, which can be reasonably attributed to the blocking of smaller particles. 


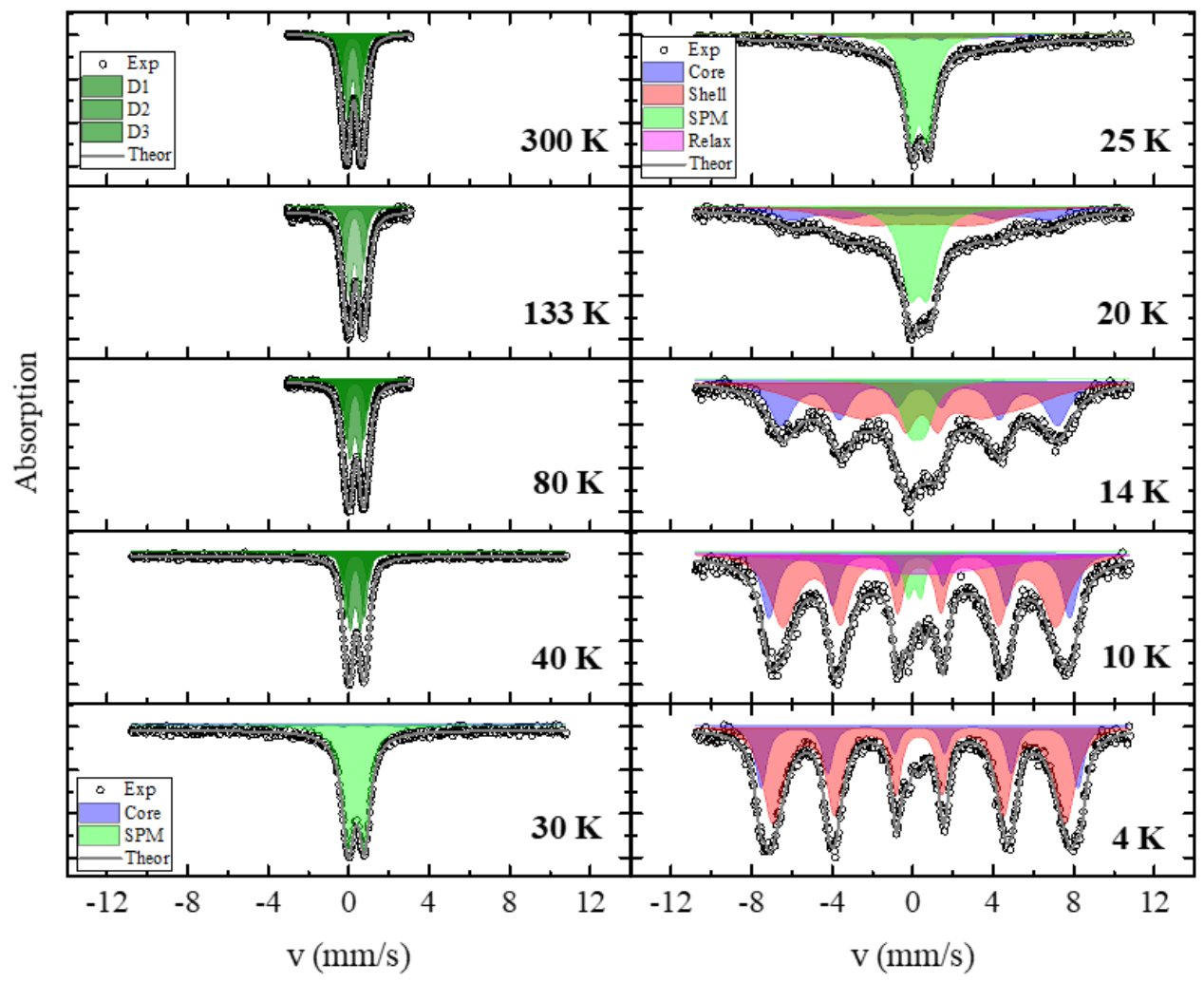

Fig. 3. Mössbauer spectra of the biogenic ferrihydrite sample at different temperatures within the range of 4-300 K. The partial components obtained by processing of the spectra are shown (see the text and Table 1).

We note that, in some cases, the transition from the SP to blocked state is accompanied by the occurrence of a relaxation component in the Mössbauer spectra of nanoparticle systems, which can be approximated by a single broad line $[9,58]$. In our case, its contribution is negligible (see Table 1 and Fig. 3). This can be explained by the absence of interparticle magnetic interactions, which are prevented by the polysaccharide shell of biogenic ferrihydrite nanoparticles [44, 57, 59]. This makes it possible to reliably distinguish the SP spectral component (doublet) in the temperature range of 4 $30 \mathrm{~K}$ against the background of particles with a blocked magnetic moment, which are reflected by a sextet with broadened lines. The temperature dependences of the fractions of the magnetically split (sextet) and SP (doublet) spectral components are shown in Fig. 4. Due to the particle size distribution, the hyperfine structure of the Mössbauer spectra smoothly collapses. A fairly narrow (4-30 K) temperature range (as compared, for example, with the results of $[58,60,61]$ ) of the transition from the blocked to SP state follows from the narrow size distribution of the investigated ferrihydrite nanoparticles (Fig. 1). 


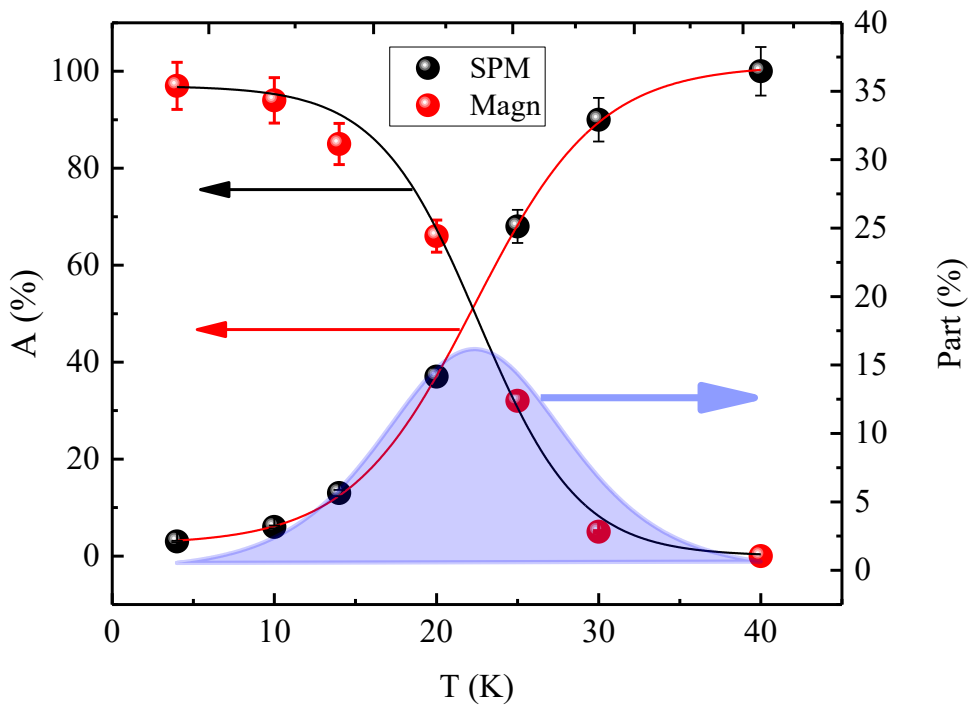

Fig. 4. Temperature dependences of the populations of the doublet (SP) and magnetically split (sextet - Magn) components of the Mössbauer spectrum. The fill area shows the blocking temperature distribution of nanoparticles.

Let us consider the process of nanoparticle blocking in more detail using the Mössbauer spectrometry and magnetic measurement data. The magnetic moment of nanoparticles of a certain size is blocked at a specific temperature, which can be determined by the Néel-Brown relationship

$$
\mathrm{T}_{\mathrm{B}}=\mathrm{K}_{\mathrm{eff}} \mathrm{V} / \ln \left(\tau / \tau_{0}\right) k_{\mathrm{B}}
$$

In this equation, $\mathrm{K}_{\mathrm{eff}}$ is the effective magnetic anisotropy constant, $\mathrm{V}$ is the particle volume, $\tau_{0}$ is the characteristic particle relaxation time lying conventionally between $10^{-13}-10^{-9} \mathrm{~s}$ [8], and $\tau$ is the characteristic measuring time, which is $\sim 10^{2} \mathrm{~s}$ for the quasi-static magnetic measurements, while in Mössbauer spectroscopy, is determined by the characteristic lifetime of ${ }^{57} \mathrm{Fe}$ nuclei in the excited state and amounts to $\sim 2.5 \cdot 10^{-8} \mathrm{~s}$ [62]. The effective magnetic anisotropy is frequently expressed as [63-65]

$$
\mathrm{K}_{\mathrm{eff}}=\mathrm{K}_{\mathrm{V}}+6 \mathrm{~K}_{\mathrm{S}} / \mathrm{d}
$$

Equation (2) takes into account the contributions of the bulk $\left(\mathrm{K}_{\mathrm{V}}\right)$ and surface magnetic anisotropy. The latter is induced by the large fraction of spins with the incomplete environment $[66,67]$ and can be characterized by the surface anisotropy constant $\mathrm{K}_{\mathrm{s}}$.

The magnetic measurements illustrated in Fig. $2 \mathrm{~b}$ yield the blocking temperature distribution function $f\left(\mathrm{~T}_{\mathrm{B}}\right) \sim-\left(\mathrm{d}\left(\mathrm{M}(\mathrm{T})_{\mathrm{FC}}-\mathrm{M}(\mathrm{T})_{\mathrm{ZFC}}\right) / \mathrm{dT}\right.$. In the Mössbauer spectroscopy, the temperature dependence of the SP component fraction (Fig. 4) can be described by the variable probability integral $[58,68]$ 


$$
\Phi(x)=\frac{2}{\sqrt{2 \pi}} \int_{s=1}^{4^{N}} e^{-\frac{x^{2}}{2}} d t .
$$

Here, $\Phi(\mathrm{x})$ is the blocking temperature distribution function: $\Phi(\mathrm{x}) \infty \sim f\left(\mathrm{~T}_{\mathrm{B}}\right)$ (Fig. 4). Obviously, the average values $\left\langle\mathrm{T}_{\mathrm{B}}\right\rangle$ and the distribution functions $f\left(\mathrm{~T}_{\mathrm{B}}\right)$ (Figs. $2 \mathrm{~b}$ and 4 ) obtained by different experimental techniques are different because of the difference in the characteristic times $\tau$, according to Eq. (1). However, at the correct $\mathrm{K}_{\mathrm{eff}}$ and $\tau_{0}$ values, the recalculation of the $f\left(\mathrm{~T}_{\mathrm{B}}\right)$ dependences using Eq. (1) in the $f(\mathrm{~d})$ distribution function should yield the coincidence of the data obtained by different techniques, as well as the coincidence with the distribution based on the TEM data (Fig. 1).

Using Eqs. (1) and (2), we obtain

$$
\mathrm{KVd}^{3}+6 \mathrm{~K}_{\mathrm{S}} \mathrm{d}^{2}=\ln \left(\tau / \tau_{0}\right) k_{\mathrm{B}} T_{\mathrm{B}}
$$

Solving numerically cubic equation (4), we can obtain the particle size distribution $f(\mathrm{~d})$ from the $f\left(\mathrm{~T}_{\mathrm{B}}\right)$ dependence. The best agreement between the calculated $f(\mathrm{~d})$ dependences obtained from the magnetic measurements and Mössbauer spectroscopy data and the TEM results was obtained at $\mathrm{K}_{V}=1.2$. $10^{5} \mathrm{erg} / \mathrm{cm}^{3}, \mathrm{~K}_{\mathrm{S}}=0.1 \mathrm{erg} / \mathrm{cm}^{2}$, and $\tau_{0}=10^{-12} \mathrm{~s}$. Figure 5 shows the calculated $f(\mathrm{~d})$ dependences along with the TEM data. It can be seen that these dependences agree well with each other. It is noteworthy that the use of Eq. (1) and the effective anisotropy constant for average-size particles $\left(\mathrm{K}_{\mathrm{eff}}(<\mathrm{d}\rangle=\right.$ $2 \mathrm{~nm}) \approx 3.1 \cdot 10^{6} \mathrm{erg} / \mathrm{cm}^{3}$ ) noticeably narrow the bulb of the calculated $f(\mathrm{~d})$ dependences. The results obtained confirm the need for taking into account the surface anisotropy contribution in ferrihydrite, similar to other oxide nanoparticles $[33,69-71]$. The $\mathrm{K}_{\mathrm{V}}$ and $\mathrm{K}_{\mathrm{S}}$ values are consistent with the results obtained for ferrihydrite in $[41,43,55]$.

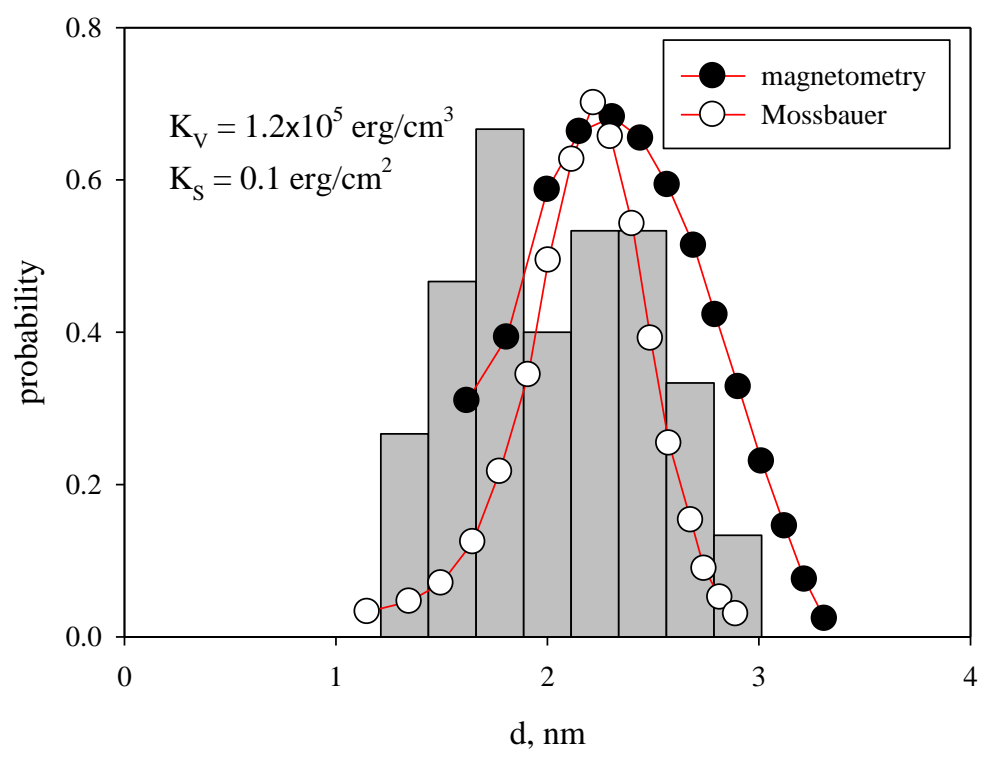


Fig. 5. Calculated ferrihydrite nanoparticle size distributions determined by Mössbauer spectroscopy (open symbols) and magnetometry (closed symbols) and from the TEM data (histogram).

\subsection{Manifestation of the Core-Shell Structure in the Mössbauer Spectra}

Thus, according to the Mössbauer spectroscopy data, the magnetic moments of particles are blocked in the temperature range from 30 to $4 \mathrm{~K}$. In the SP state (T > $30 \mathrm{~K}$ ), the doublet corresponds to three nonequivalent positions of ferric iron in the octahedral environment characteristic of ferrihydrite (Table 1). However, during the transition to the blocked state, the magnetically split part of the spectrum (sextet) should reflect the features characteristic of the inhomogeneous structure of the nanoparticles themselves [7]. In this approximation, the Mössbauer spectrum for the blocked state can consist of at least two subspectra S1 and S2, which should represent the averaged parameters of nonequivalent magnetic states of iron [7, 57]: S1 in the core and S2 in the shell. This approach was taken into account during processing of the magnetically split spectral component in the temperature range of 4-30 K. The results of the spectra processing based on this model representation are given in Table 1; the temperature evolution of the populations of the corresponding subspectra is shown in the inset in Fig. 6. It is noteworthy that the population of spectrum component S1 responsible for iron atoms in the particle core remains almost unchanged in the range of 4-25 K, but the fraction of sextet S2, which is taken by us for the surface states of iron atoms, changes quite strongly. This can be related to the fact that, with increasing temperature, we have a complex transition of particles to the SP state, which, in addition to the effect of the particle size distribution (Fig. 5), reflects the features of the inhomogeneous structure of ferrihydrite particles.

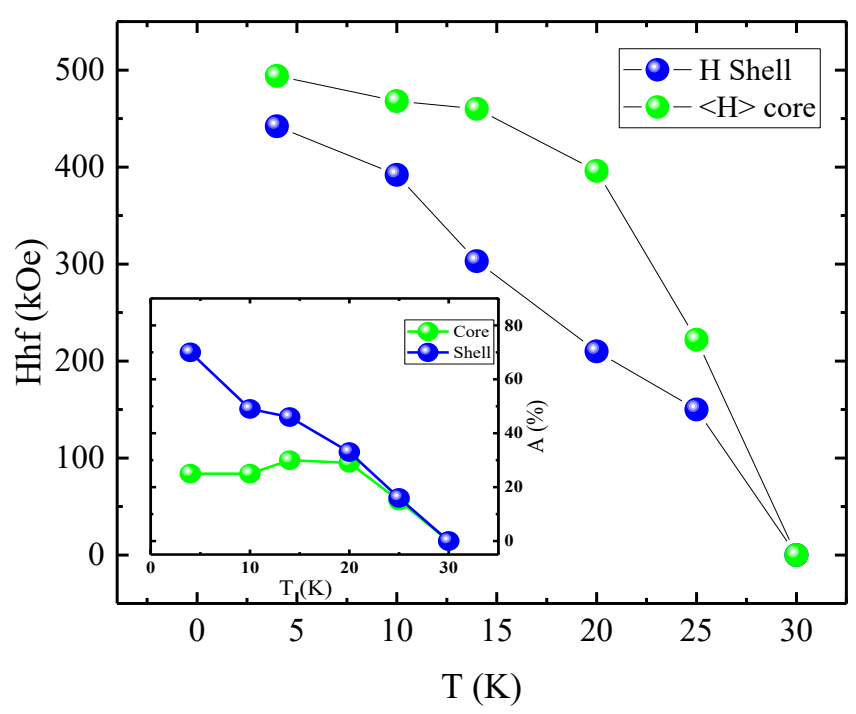


Fig. 6. Temperature dependences of populations A (the ordinate axis is the lefthand scale) and hyperfine field $\mathrm{H}_{\mathrm{hf}}$ (the ordinate axis is the right-hand scale) of internal states S1 (core) and surface states S2 (shell) of ${ }^{57} \mathrm{Fe}$ in the investigated ferrihydrite obtained by processing of the Mössbauer spectra.

The differences between the formation of the core and surface magnetic structures can be clearly seen in the temperature dependence of the hyperfine fields $\mathrm{H}_{\mathrm{hf}}(\mathrm{T})$ (Fig. 6). On the particle surface, the bond breaking occurs, which usually leads to the reduction of the iron coordination and, in the hydrated state, to the formation of new bonds upon filling of coordination spheres on the surface. Hence, the hyperfine parameters (hyperfine field) of ${ }^{57} \mathrm{Fe}$ nuclei for sextet S2 appear somewhat underestimated due to the uneven arrangement of magnetic neighbors as compared with those in the particle core (S1). The magnetic correlation radius decreases with increasing temperature; one can see that a decrease in the hyperfine field is more pronounced for the iron surface states, which can be reasonably attributed to the weakening of magnetic interactions with increasing distance from the particle center.

Let us consider the temperature evolution of the ratio $A_{S 2} / A_{S 1}$ between the populations of subspectra $S 2$ and $\mathrm{S} 1$ (see the inset in Fig. 7). The $\mathrm{A}_{\mathrm{S} 2} / \mathrm{A}_{\mathrm{S} 1}$ dependence is a linear function of temperature. This, in fact, is the ratio between volumes $\mathrm{V}_{\text {shell }}$ of the surface states and volumes $\mathrm{V}_{\text {core }}$ of the core for all particles blocked at a specific temperature: $\mathrm{A}_{\mathrm{s} 2} / \mathrm{A}_{\mathrm{S} 1} \approx \mathrm{V}_{\text {shell }} / \mathrm{V}_{\text {core }}$ at $\mathrm{T}=$ const. From general considerations, we can write

$$
\frac{V_{\text {core }}}{V_{\text {shell }}} \approx \frac{R^{3}}{(R+\Delta r)^{3}-R^{3}} .
$$

Here, $\Delta r$ is the thickness of the surface (loose) particle layer and $R$ is the radius of the dense particle core; obviously, $2(\mathrm{R}+\Delta r)=\mathrm{d}$. Knowing the value in the left-hand side of Eq. (5) from the $\mathrm{A}_{\mathrm{S} 2} / \mathrm{A}_{\mathrm{S} 1}$ data (inset in Fig. 7), as well as the blocking temperature distribution functions $f\left(\mathrm{~T}_{\mathrm{B}}\right)$ (Fig. 4) and the size distribution functions $f(\mathrm{~d})$ (Fig. 5), we can obtain the $\mathrm{R}$ and $\Delta r$ values as functions of the total particle diameter $d$. It was found that, for a particle with the maximum size $(d \approx 3 \mathrm{~nm})$, the surface layer thickness $\Delta r$ is smaller than $0.3 \mathrm{~nm}$. As the particle size decreases, the ratio between the volumes of the dense core and the porous surface decreases. For example, in a particle with a diameter of $\mathrm{d} \approx 2.2 \mathrm{~nm}$, we have $\Delta r \sim 0.45 \mathrm{~nm}$ at $2 \mathrm{R} \approx 1.3 \mathrm{~nm}$, whereas, in the case of particles with sizes d smaller than two nanometers, we can no longer speak about the dense core and, in fact, over the entire particle volume, the states of iron are similar to those of the porous surface. The aforesaid is illustrated in Fig. 7, which shows the dependence of the size of the dense core of ferrihydrite particles (in our designations, 2R) on the particle diameter $\mathrm{d}$. In addition, the figure shows the proposed model 
representation of ferrihydrite nanoparticles of different sizes with the distinguished regions of different densities (different states of iron atoms, according to the Mössbauer spectroscopy data).

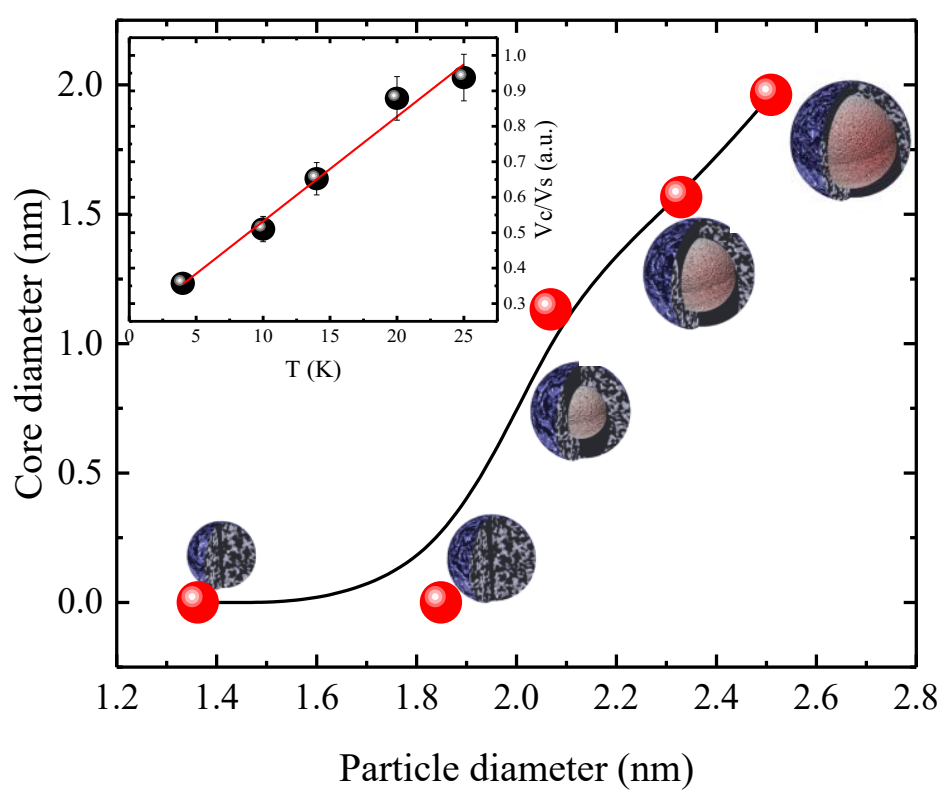

Fig. 7. Dependence of the ferrihydrite particle core size on the particle diameter. Inset: temperature dependence of the ratio between the volumes of the particle core and surface states according to the Mössbauer spectroscopy data.

It was obtained in [16] by analyzing the thermodynamic stability of ferrihydrite particles that, at a particle size of smaller than $1.5 \mathrm{~nm}$, the amount of Gibbs free energy spent to the formation of the surface $\left(\sim \mathrm{R}^{2}\right)$ is larger than the gain resulting from the nucleation $\left(\sim \mathrm{R}^{3}\right)$. In other words, such particles are kind of surface aggregates with the branched surface and without bulk crystalline core. This is consistent with our results, according to which a crystalline core starts forming only at a particle size of larger than $2 \mathrm{~nm}$ (Fig. 5). In this case, both the dense and porous phases exhibit the stable thermochemical properties [16]. Thus, ferrihydrite is a heterogeneous formation in which, after exceeding a certain particle size, a dense crystalline core is formed. Such a particle growth model determines the magnetic properties of the investigated material.

\section{Conclusions}

The biogenic ferrihydrite samples were formed by the activity of the Klebsiella oxytoca bacteria. Using the TEM investigations, the average size of the nanoparticles was found to be $2 \mathrm{~nm}$. The Mössbauer effect and the magnetic properties of the obtained samples were measured in the temperature range of 4-300 K. The analysis of the blocking/unlocking of the particle magnetic 
moment showed the dominating role of the surface magnetic anisotropy contribution to these processes and allowed us to calculate the bulk and surface magnetic anisotropy constants $\mathrm{K}_{\mathrm{V}}$ and $\mathrm{K}_{\mathrm{S}}\left(\mathrm{K}_{\mathrm{V}}=1.2\right.$. $10^{5} \mathrm{erg} / \mathrm{cm}^{3}$ and $\mathrm{K}_{\mathrm{S}}=0.1 \mathrm{erg} / \mathrm{cm}^{2}$ ). Using these data, the particle size distribution curve was calculated and appeared to be in good agreement with the TEM data.

It was experimentally shown that the magnetic properties of ferrihydrite nanoparticles are caused by the formation of core-shell systems. In the first approximation, these states correspond to the surface (loose) layer and the well-crystallized dense core of a particle. According to the Mössbauer spectroscopy data, such a model is valid for particles larger than $2 \mathrm{~nm}$. This size is critical for the formation of a well-crystallized core in a ferrihydrite particle, whereas smaller particles consist entirely of a material with the lower density. Thus, the contribution of the surface to the magnetic properties of ferrihydrite appears to be variable and depends on both the total particle size and the ratio between the surface fraction and the particle volume.

\section{Acknowledgments}

The electron microscopy study was carried out on the equipment of the Krasnoyarsk Territorial Center for Collective Use, Krasnoyarsk Scientific Center, Siberian Branch, Russian Academy of Sciences.

\section{Funding}

This study was supported by the Russian Foundation for Basic Research, the Government of the Krasnoyarsk Territory, and the Krasnoyarsk Territorial Foundation for Support of Scientific and R\&D Activities, project no. 19-42-240012 p_a "Magnetic Resonance in Ferrihydrite Nanoparticles: Effects Related to the Core-Shell Structure".

[1] J. van der Geer, J.A.J. Hanraads, R.A. Lupton, The art of writing a scientific article, J. Sci. Commun. 163 (2010) 51-59. https://doi.org/10.1016/j.Sc.2010.00372.

Reference to a book:

[3] W. Strunk Jr., E.B. White, The Elements of Style, fourth ed., Longman, New York, 2000. Reference to a chapter in an edited book:

[1] U. Schwertmann, J. Friedl, H. Stanjek, From Fe (III) ions to ferrihydrite and then to hematite J. Colloid Interface Sci., 209 (1999) 215-223. https://doi.org/10.1006/jcis.1998.5899

[2] T. Hiemstra, Surface structure controlling nanoparticle behavior: magnetism of ferrihydrite, magnetite, and maghemite, Environ. Sci.: Nano, 5 (2018) 752-764. https://doi.org/10.1039/C7EN01060E 
[3] J. L. Bishop, C. M. Pieters, R. G. Burns, Reflectance and Mössbauer spectroscopy of ferrihydritemontmorillonite assemblages as Mars soil analog materials, Geochim. Cosmochim. Acta, 57 (1993), 4583-4595. https://doi.org/10.1016/0016-7037(93)90184-X

4. G. Klingelhöfer1, R. V. Morris, B. Bernhardt, C. Schröder, D. S. Rodionov, P. A. de Souza Jr., A. Yen, R. Gellert, Jarosite and Hematite at Meridiani Planum from Opportunity's Mössbauer Spectrometer, Science, 306 (2004), 1740-1745. https://doi.org/10.1126/science.1104653

5. B. D. Kocar, T. Borch, S. Fendorf, Arsenic repartitioning during biogenic sulfidization and transformation of ferrihydrite, Geochim. Cosmochim. Acta, 74 (2010) 980-994. https://doi.org/10.1016/j.gca.2009.10.023

6. M. Balasoiu, S.V. Stolyar, R. S. Iskhakov, L.A. Ishchenko, Yu.L. Raikher, A.I. Kuklin, O.L. Orelovich, Yu.S. Kovalev, T.S. Kurkin, G.M. Arzumanian, Hierarchical Structure Investigations Of Biogenic Ferrihydrite Samples, Rom. Journ. Phys., 55 (2010) 782-789.

7. G.C. Papaefthymiou, The Mössbauer and magnetic properties of ferritin cores, Biochim. Biophys. Acta 1800 (2010) 886-897. https://doi.org/10.1016/j.bbagen.2010.03.018

8. S. Mørup, D.E. Madsen, C. Fradsen, C.R.H. Bahl, M.F. Hansen, 2007. Experimental and theoretical studies of nanoparticles of antiferromagnetic materials, J. Phys.: Condens. Matter. 19213202. https://doi.org/10.1088/0953-8984/19/21/213202

9. E. Murad, L. H. Bowen, G. J. Long and T. G. Quin, The influence of crystallinity on magnetic ordering in natural ferrihydrites Clay Minerals 23 (1988) 161-173. https://doi.org/10.1180/claymin.1988.023.2.04

10. E.Lima Jr., A.D.Arelaro, H.R.Rechenberg, E.L.Duarte, R.Itri, C.Cavelius, H.Shen, S.Mathur, G.F.Goya, Magnetic characterization of ferrihydrite nanoparticles synthesized by hydrolysis of Fe metal-organic precursor, Physica B: Cond. Mat., $403 \quad$ (2008) 4156-4159. https://doi.org/10.1016/j.physb.2008.08.018

11. Z. Homonnay, Gy. Tolnai, F. Fodor, Á. Solti, K. Kovács, E. Kuzmann, A. Ábrahám, E. Gy. Szabó, P. Németh, L. Szabó and Z. Klencsár, Iron oxide nanoparticles for plant nutrition? A preliminary Mössbauer study, Hyperfine Interactions. 237 (2016) 127. https://doi.org/10.1007/s10751-016-1334-1 12. F. M. Michel, L. Ehm, S. M. Antao, P. L. Lee, P. J. Chupas, G. Liu, D. R. Strongin, M. A. A. Schoonen, B. L. Phillips, J. B. Parise, The Structure of Ferrihydrite, a Nanocrystalline Material, Science 316 (2007), 1726-1729. https://doi.org/10.1126/science.1142525

13. D.G. Rancourt, J.-F. Meunier, Constraints on structural models of ferrihydrite as a nanocrystalline material, Am. Mineral., 93 (2008) 1412-1417, 2008. https://doi.org/10.2138/am.2008.2782

14. V. M. Yuwono, N. D. Burrows, J. A. Soltis, T. A. Do and R. L. Penn, Aggregation of ferrihydrite nanoparticles in aqueous systems, Faraday Discuss., $159 \quad$ (2012) 235-245. https://doi.org/10.1039/C2FD20115A

15 T. Hiemstra, Surface and mineral structure of ferrihydrite, Geochim. Cosmochim. Acta,, 10 (2013) 316-325. https://doi.org/10.1016/j.gca.2012.12.002 
16 T. Hiemstra, Formation, stability, and solubility of metal oxide nanoparticles: Surface entropy, enthalpy, and free energy of ferrihydrite, Geochim. Cosmochim. Acta,, 158 (2015) 179-198. https://doi.org/10.1016/j.gca.2015.02.032

17. P. G. Weidler, H. Stanjek, The effect of dry heating of synthetic 2-line and 6-line ferrihydrite: II. Surface area, porosity and fractal dimension, Clay Miner., 33 (1998) 277-284. https://doi.org/10.1180/000985598545471

18. B. A. Legg, M. Zhu, L. R. Comolli, B. Gilbert, J. F. Banfield, Determination of the threedimensional structure of ferrihydrite nanoparticle aggregates, Langmuir, 30 (2014) 9931-9940. https://doi.org/10.1021/la502128d

19 M.S. Seehra, V.S. Babu, A. Manivannan, J.W. Lynn, Neutron scattering and magnetic studies of ferrihydrite nanoparticles, Phys. Rev. B 61 (2000) 3513. https://doi.org/10.1103/PhysRevB.61.3513

20 S.A. Makhlouf, F.T. Parker, F.E. Spada, and A.E. Berkowitz, Magnetic anomalies in NiO nanoparticles, J. Appl. Phys. 81 (1997) 5561. https://doi.org/10.1063/1.364661

21 J.G.E. Harris, J.E. Grimaldi, D.D. Awschalom, A. Chilero, and D. Loss, Excess spin and the dynamics of antiferromagnetic ferritin, Phys. Rev. B 60 (1999) 3513. https://doi.org/10.1103/PhysRevB.60.3453

22 A. Punnoose, T. Phanthavady, M.S. Seehra, N. Shah, and G.P. Huffman, Magnetic properties of ferrihydrite nanoparticles doped with Ni, Mo, and Ir, Phys. Rev. B 69 (2004) 054425. https://doi.org/10.1103/PhysRevB.69.054425

23 N.J.O. Silva, V.S. Amaral, L.D. Carlos, Relevance of magnetic moment distribution and scaling law methods to study the magnetic behavior of antiferromagnetic nanoparticles: Application to ferritin, Phys. Rev. B 71 (2005) 184408 (2005). https://doi.org/10.1103/PhysRevB.71.184408

24 M.S. Seehra, V. Singh, X. Song, S. Bali, E.M. Eyring, Synthesis, structure and magnetic properties of non-crystalline ferrihydrite nanoflakes, J. Phys. Chem. Solids 71 (2010) 1362. https://doi.org/10.1016/j.jpcs.2010.06.003

25 D.A. Balaev, A.A. Dubrovskii, A.A. Krasikov, S.V. Stolyar, R.S. Iskhakov, V.P. Ladygina, E.D. Khilazheva, Mechanism of the formation of an uncompensated magnetic moment in bacterial ferrihydrite nanoparticles, JETP Lett. 98 (2013) 135. https://doi.org/10.1134/S0021364013160029

26 D.A. Balaev, A.A. Krasikov, A.A. Dubrovskii, S.V. Semenov, O.A. Bayukov, S. V. Stolyar, R.S. Iskhakov, V.P. Ladygina, L.A. Ishchenko, Magnetic properties and the mechanism of formation of the uncompensated magnetic moment of antiferromagnetic ferrihydrite nanoparticles of a bacterial origin, JETP 119 (3), 479 (2014). https://doi.org/10.1134/S1063776114080044

27 Yu.L. Raikher, V.I. Stepanov, Magneto-orientational behavior of a suspension of antiferromagnetic particles, J. Phys.: Condens. Matter 20 (2008) 204120. https://doi.org/10.1088/0953$\underline{8984 / 20 / 20 / 204120}$ 
28 S.D. Tiwari, K.P. Rajeev, Effect of distributed particle magnetic moments on the magnetization of $\mathrm{NiO}$ nanoparticles, Solid State Commun. 152 (2012) 1080. https://doi.org/10.1016/j.ssc.2012.03.003

29 Ch. Rani, S.D. Tiwari, Superparamagnetic behavior of antiferromagnetic six lines ferrihydrite nanoparticles, Physica B 513 (2017) 58. https://doi.org/10.1016/j.physb.2017.02.036

30 Ch. Rani, S.D. Tiwari, Estimation of particle magnetic moment distribution for antiferromagnetic ferrihydrite nanoparticles, J. Magn. Magn. Mater. $385 \quad$ (2015) 272. https://doi.org/10.1016/j.jmmm.2015.02.048

31 A.A. Lepeshev, I.V. Karpov, A.V. Ushakov, D.A. Balaev, A.A. Krasikov, A.A. Dubrovskiy, D.A. Velikanov, M.I. Petrov, Particularities of the Magnetic State of CuO Nanoparticles Produced by Low-Pressure Plasma Arc Discharge, J. Supercond. Nov. Magn. 30 (2017) 931. https://doi.org/10.1007/s10948-016-3885-4

32 Ch. Parmar, G.S. Parmar, Structural and Magnetic Properties of Six-Line Ferrihydrite Nanoparticles, J. Supercond. Nov. Magn. 33 (2020) 441. https://doi.org/10.1007/s10948-019-05200-X 33 S.I. Popkov, A.A. Krasikov, D.A. Velikanov, V.L. Kirillov, O.N. Martyanov, D.A. Balaev, Formation of the magnetic subsystems in antiferromagnetic $\mathrm{NiO}$ nanoparticles using the data of magnetic measurements in fields up to 250 kOe, J. Magn. Magn. Mater. 483 (2019) 21. https://doi.org/10.1016/j.jmmm.2019.03.004

34 S.I. Popkov, A.A. Krasikov, A.A. Dubrovskiy, M.N. Volochaev, V.L. Kirillov, O.N. Martyanov, D.A. Balaev, Size effects in the formation of an uncompensated ferromagnetic moment in $\mathrm{NiO}$ nanoparticles, J. Appl. Phys. 126 (2019) 103904. https://doi.org/10.1063/1.5109054

35 T. Iimori, Y. Imamoto, N. Uchida, Y. Kikuchi, K. Honda, T. Iwahashi, Y. Ouchi, Magnetic moment distribution in nanosized antiferromagnetic NiO, J. Appl. Phys. 127 (2020) 023902. https://doi.org/10.1063/1.5135335

36 P. Dutta, S. Pal, M.S. Seehra, N. Shah, G.P. Huffman, Size dependence of magnetic parameters and surface disorder in magnetite nanoparticles, J. Appl. Phys. 105 (2009) 07B501. https://doi.org/10.1063/1.3055272

37 A. Millan, A. Urtizberea, N.J.O. Silva, F. Palacio, V.S. Amaral, E. Snoeck, V. Serin, Surface effects in maghemite nanoparticles, J. Magn. Magn. Mater. 312 (2007) L5. https://doi.org/10.1016/j.jmmm.2006.09.011

38 V.L. Kirillov, D.A. Balaev, S.V. Semenov, K.A. Shaikhutdinov, O.N. Martyanov, Size control in the formation of magnetite nanoparticles in the presence of citrate ions, Mater. Chem. Phys. 145 (2014) 75. https://doi.org/10.1016/j.matchemphys.2014.01.036

39 K. Dobretsov, S. Stolyar, A. Lopatin, Magnetic nanoparticles: a new tool for antibiotic delivery to sinonasal tissues. Results of preliminary studies, Acta Otorhinolaryngol. Ital. 35 (2015) 97-102. 
40 S.V. Stolyar, D.A. Balaev, V.P. Ladygina, A.A. Dubrovskiy, A.A. Krasikov, S.I. Popkov, O.A. Bayukov, Yu.V. Knyazev, R.N. Yaroslavtsev, M.N. Volochaev, R.S. Iskhakov, K.G. Dobretsov, E.V. Morozov, O. V.Falaleev, E. V. Inzhevatkin, O.A. Kolenchukova, I.A. Chizhova, Bacterial ferrihydrite nanoparticles: preparation, magnetic properties, and application in medicine, J. Supercond. Nov. Magn. 31 (2018) 2297. https://doi.org/10.1007/s10948-018-4700-1

41 E.L. Duarte, R. Itri, E. Lima Jr, M.S. Baptista, T.S. Berquó, G.F. Goya, Large magnetic anisotropy in ferrihydrite nanoparticles synthesized from reverse micelles, Nanotechnology 17 (2006) 5549. https://doi.org/10.1088/0957-4484/17/22/004

42 H. Shim, P. Dutta, M.S. Seehra, J. Bonevich, Size dependence of the blocking temperatures and electron magnetic resonance spectra in NiO nanoparticles, Solid State Commun. 145 (2008) 192-196. https://doi.org/10.1016/j.ssc.2007.10.026

43 T.S. Berquó, J.J. Erbs, A. Lindquist, R.L. Penn, S.K. Banerjee, Effects of magnetic interactions in antiferromagnetic ferrihydrite particles, J. Phys.: Condens. Matter. 21 (2009) 176005. https://doi.org/10.1088/0953-8984/21/17/176005

44 S.V. Stolyar, D.A. Balaev, V.P. Ladygina, A.I. Pankrats, R.N. Yaroslavtsev, D.A. Velikanov, R.S. Iskhakov, JETP Letters 111, 183 (2020).

45 L. Anghel, M. Balasoiu, L.A. Ishchenko, S.V. Stolyar, T.S. Kurkin, A.V. Rogachev, A.I. Kuklin, Y. S. Kovalev, Y.L. Raikher, R.S. Iskhakov, and G. Duca, Characterization of bio-synthesized nanoparticles produced by Klebsiella oxytoca, J. Phys. Conf. Ser. 351 (2012) 12005. doi:10.1088/1742-6596/351/1/012005

46 S.V. Stolyar, O.A. Bayukov, D.A. Balaev, R. S. Iskhakov, L.A. Ishchenko, V.P. Ladygina, and R.N. Yaroslavtsev, Production and magnetic properties of biogenic ferrihydrite nanoparticles, J. Optoelectron. Adv. Mater. 17 (2015) 968.

47 V.P. Ladygina, K.V. Purtov, S.V. Stoljar, R. S. Iskhakov, O.A. Bajukov, J. L. Gurevich, K.G. Dobretsov, and L.A. Ishchenko, Method of producing stable aqueous sol based on ferrihydrite nanoparticles: pat. EA018956. Russia (2013).

48 D.A.Velikanov, SQUID magnetometer for investigations of the magnetic properties of materials in the temperature range 4.2-370 K, Sib. J. Sci. Technol. 2 (2013) 176-181.

49 R.K. Kukkadapu, J.M. Zachara, J.K. Fredrickson, S.C. Smith, A. C. Dohnalkova, C. K. Russell. Transformation of 2-line ferrihydrite to 6-line ferrihydrite under oxic and anoxic conditions, Am. Mineral. 88 (2003) 1903. https://doi.org/10.2138/am-2003-11-1233

50 Y. Guyodo, S.K. Banerjee, R. Lee Penn, D. Burleson, T.S. Berquo, T. Seda, P. Solheid, Magnetic properties of synthetic six-line ferrihydrite nanoparticles, Phys. Earth Planet. Inter. 154 (2006) 222. https://doi.org/10.1016/j.pepi.2005.05.009

51 S.V. Stolyar, R.N. Yaroslavtsev, R.S. Iskhakov, O.A. Bayukov, D.A. Balaev, A.A. Dubrovskii, A.A. Krasikov, V.P. Ladygina, A.M. Vorotynov, M.N. Volochaev, Magnetic and resonance properties of ferrihydrite nanoparticles doped with cobalt, Phys. Solid State 59 (2017) 555. https://doi.org/10.1134/S1063783417030301 
52 J.C. Denardin, A.L. Brandl, M. Knobel, P. Panissod, A.B. Pakhomov, H. Liu, X.X. Zhang. Thermoremanence and zero-field-cooled/field-cooled magnetization study of $\mathrm{Co}\left(\mathrm{SiO}_{2}\right)$ granular films, Phys. Rev. B 65 (2002) 064422. https://doi.org/10.1103/PhysRevB.65.064422

53 D. Tobia, E. Winkler, R.D. Zysler, M. Granada, H.E. Troiani, D. Fiorani. Exchange bias of Co nanoparticles embedded in $\mathrm{Cr}_{2} \mathrm{O}_{3}$ and $\mathrm{Al}_{2} \mathrm{O}_{3}$ matrices, J. Appl. Phys. 106 (2009) 103920. https://doi.org/10.1063/1.3259425

54 D.A. Balaev, A.A. Krasikov, S.V. Stolyar, R.S. Iskhakov, V.P. Ladygina, R.N. Yaroslavtsev, O.A. Bayukov, A.M. Vorotynov, M.N. Volochaev, A.A. Dubrovskii, Change in the magnetic properties of nanoferrihydrite with an increase in the volume of nanoparticles during low-temperature annealing, Phys. Solid State 58 (2016) 1782. https://doi.org/10.1134/S1063783416090092

55 D.A. Balaev, A.A. Krasikov, A.A. Dubrovskiy, S.I. Popkov, S.V. Stolyar, O.A. Bayukov, R.S. Iskhakov, V.P. Ladygina, R.N. Yaroslavtsev. Magnetic properties of heat treated bacterial ferrihydrite nanoparticles, J. Magn. Magn. Mater. 410 (2016) 71. https://doi.org/10.1016/j.jmmm.2016.02.059

56. T.G.St. Pierre, P.Chan, K.R.Bauchspiess, J.Webb, S.Betteridge, S.Walton, D.P.E.Dickson, Synthesis, structure and magnetic properties of ferritin cores with varying composition and degrees of structural order: models for iron oxide deposits in iron-overload diseases, Coord. Chem. Rev.. 151 (1996) 125-143. https://doi.org/10.1016/S0010-8545(96)90201-5

57 F.B. Abdallah, E. Carney, N. D. Chasteen, P. Arosio, A. J. Viescas, G. C. Papaefthymiou, A comparative Mössbauer study of the mineral cores of human H-chain ferritin employing dioxygen and hydrogen peroxide as iron oxidants, Biophys. Chem. $130 \quad$ (2007) 114-121. https://doi.org/10.1016/j.bpc.2007.08.003

58 Yu. V. Knyazev, D. A. Balaev, V. L. Kirillov, O. A. Bayukov and O. N. Mart'yanov, Mössbauer Spectroscopy Study of the Superparamagnetism of Ultrasmall $\epsilon-\mathrm{Fe}_{2} \mathrm{O}_{3}$ Nanoparticles, JETP Lett. 108 (2018): 527-531. https://doi.org/10.1134/S0021364018200092

59 Bonville, C. Gilles, Search for incoherent tunnel fluctuations of the magnetisation in nanoparticles of artificial ferritin, Physica B: Cond. Matt. 304 (2001) 237-245. https://doi.org/10.1016/S0921$\underline{4526(01) 00540-3}$

60 Kündig W. H. Bömmel, G. Constabaris, and R. H. Lindquist. Some Properties of Supported Small $\alpha-\mathrm{Fe}_{2} \mathrm{O}_{3}$ Particles Determined with the Mössbauer Effect, Phys. Rev. 142 (1966) 327. https://doi.org/10.1103/PhysRev.142.327

61 Kuhn, L. T., Lefmann, K., Bahl, S. N. Ancona, P.-A. Lindgård, C. Frandsen, D. E. Madsen, and S. Mørup, Neutron study of magnetic excitations in $8 \mathrm{~nm} \alpha-\mathrm{Fe}_{2} \mathrm{O}_{3}$ nanoparticles, Phys. Rev. B, 74 (2006).184406. https://doi.org/10.1103/PhysRevB.74.184406

62 I. P. Suzdalev, O superparamagnetizme ul'tramalykh chastits antiferromagnetikov, Solid State Phys. 12 (1970) 988 (in Russian).

63 A. Aharoni, Surface anisotropy in micromagnetics, J. Appl. Phys. 61 (1987) 3302-3304. https://doi.org/10.1063/1.338890 
64 F. Bødker, S. Mørup, and S. Linderoth, Surface effects in metallic iron nanoparticles, Phys. Rev. Lett. 72 (1994) 282-285. https://doi.org/10.1103/PhysRevLett.72.282

65 I. G. Vazhenina, R. S. Iskhakov, and L. A. Chekanova, Spin-wave resonance in chemically deposited Fe-Ni films: Measuring the spin-wave stiffness and surface anisotropy constant, Phys. Solid State 60 (2018) 292. https://doi.org/10.1134/S1063783418020294

66 C. Chen, O. Kitakami, and Y. Shimada, Particle size effects and surface anisotropy in Fe-based granular films, J. Appl. Phys. 84 (1998) 2184-2188. https://doi.org/10.1063/1.368281

67 V.P. Shilov, J.-C. Bacri, F. Gazeau, F. Gendron, R. Perzynski, and Y. L. Raikher, Ferromagnetic resonance in ferrite nanoparticles with uniaxial surface anisotropy, J. Appl. Phys. 85 (1999) 66426647. https://doi.org/10.1063/1.370173

68 Mørup S., Topsøe H. Mössbauer studies of thermal excitations in magnetically ordered microcrystals, Appl. Phys. 11 (1976) C. 63-66. https://doi.org/10.1007/BF00895017

69 C.-R. Lin, R.-K. Chiang, J.-S. Wang, T.-W. Sung, Magnetic properties of monodisperse iron oxide nanoparticles, J. Appl. Phys. 99 (2006) 08N710. https://doi.org/10.1063/1.2172891

70 M.P. Proenca, C.T. Sousa, A.M. Pereira, P.B. Tavares, J. Ventura, M. Vazquez, J.P. Araujo, Size and surface effects on the magnetic properties of $\mathrm{NiO}$ nanoparticles, Phys. Chem. Chem. Phys. 13 (2011) 9561. https://doi.org/10.1039/C1CP00036E

71 D.A. Balaev, S.V. Semenov, A.A. Dubrovskiy, S.S. Yakushkin, V.L. Kirillov, O.N. Martyanov. Superparamagnetic blocking of an ensemble of magnetite nanoparticles upon interparticle interactions, J. Magn. Magn. Mater. 440 (2017) 199. https://doi.org/10.1016/j.jmmm.2016.12.046 
Table 1. Mossbauer spectra parameters. IS is the isomer shift relative to a-Fe, QS is the quadrupole splitting, Hhf is the hyperfine field, $\mathrm{W}$ is the full width of the line at half-maximum, $\mathrm{dW}$ is the degree of nonuniformity of the linewidth, and $\mathrm{A}$ is the relative fraction in the spectrum.

\begin{tabular}{|c|c|c|c|c|c|c|c|}
\hline & $\underline{\mathrm{IS}, \mathrm{mm} / \mathrm{s}}$ & $\underline{H_{\mathrm{hf}}, \mathrm{kOe}}$ & $\underline{\mathrm{QS}, \mathrm{mm} / \mathrm{s}}$ & $W, \mathrm{~mm} / \mathrm{s}$ & $d W, \mathrm{~mm} / \mathrm{s}$ & $A$, a.u. & Description \\
\hline & \pm 0.005 & \pm 2 & \pm 0.01 & \pm 0.01 & \pm 0.01 & \pm 0.03 & \\
\hline \multicolumn{8}{|l|}{$4 \mathrm{~K}$} \\
\hline S1 & 0.490 & 490 & 0.00 & 0.50 & 0.21 & 0.26 & Core \\
\hline S2 & 0.468 & 452 & 0.00 & 0.49 & 0.75 & 0.74 & Shell \\
\hline \multicolumn{8}{|l|}{$10 \mathrm{~K}$} \\
\hline S1 & 0.480 & 465 & 0.00 & 0.56 & 0.34 & 0.25 & Core \\
\hline S2 & 0.475 & 422 & 0.00 & 0.53 & 1.06 & 0.49 & Shell \\
\hline L1 & 0.680 & 0 & 0.01 & 8.88 & - & 0.21 & Shell \\
\hline D & 0.435 & - & 0.62 & 0.56 & - & 0.05 & SP \\
\hline \multicolumn{8}{|l|}{$14 \mathrm{~K}$} \\
\hline S1 & 0.475 & 426 & 0.00 & 0.79 & 0.34 & 0.35 & Core \\
\hline S2 & 0.507 & 303 & 0.00 & 0.29 & 1.06 & 0.52 & Shell \\
\hline D & 0.367 & - & 0.72 & 1.24 & - & 0.13 & SP \\
\hline \multicolumn{8}{|l|}{$20 \mathrm{~K}$} \\
\hline S1 & 0.553 & 395 & -0.24 & 0.79 & 0.74 & 0.28 & Core \\
\hline S2 & 0.419 & 216 & 0.00 & 0.29 & 1.40 & 0.33 & Shell \\
\hline D & 0.445 & - & 0.88 & 1.24 & - & 0.38 & SP \\
\hline \multicolumn{8}{|l|}{$25 \mathrm{~K}$} \\
\hline S1 & 0.480 & 264 & 0.00 & 0.79 & 2.94 & 0.16 & Core \\
\hline S2 & 0.478 & 151 & 0.00 & 3.05 & 0.00 & 0.15 & Shell \\
\hline D & 0.462 & - & 0.83 & 0.83 & - & 0.69 & $\mathrm{SP}$ \\
\hline \multicolumn{8}{|l|}{$30 \mathrm{~K}$} \\
\hline L1 & 0.423 & 0 & 0.00 & 0.42 & 2.41 & 0.06 & Core \\
\hline D & 0.476 & - & 0.83 & 0.67 & - & 0.94 & SP \\
\hline \multicolumn{8}{|l|}{$40 \mathrm{~K}$} \\
\hline D1 & 0.476 & - & 0.53 & 0.43 & - & 0.46 & SP1 \\
\hline D2 & 0.480 & - & 0.90 & 0.34 & - & 0.37 & SP2 \\
\hline D3 & 0.478 & - & 1.28 & 0.36 & - & 0.17 & SP3 \\
\hline \multicolumn{8}{|l|}{$300 \mathrm{~K}$} \\
\hline D1 & 0.360 & - & 0.53 & 0.34 & - & 0.47 & SP1 \\
\hline D2 & 0.358 & - & 0.86 & 0.32 & - & 0.35 & SP2 \\
\hline D3 & 0.354 & - & 1.23 & 0.34 & - & 0.17 & $\mathrm{SP} 3$ \\
\hline
\end{tabular}

